\title{
Improvements in SDD Efficiency - From X-ray Counts to Data
}

\section{Tara Nylese* and Jens Rafaelsen}

EDAX Inc., Materials Analysis Division, AMETEK, 91 McKee Drive, Mahwah, NJ 07430

*Tara.Nylese@Ametek.com

\begin{abstract}
Continuing advancements in window materials, detector modules, and electronics are leading to higher count rates, better light-element sensitivity, and improved energy-resolution stability over a wide range of count rates. In this article we will briefly review how the different parts of the EDS system interact, from X-rays leaving the sample to the production of useful data and where recent changes have taken place. We then apply the gains offered by this new technology to three samples to illustrate the benefits that can be reaped.
\end{abstract}

\section{Introduction}

The $\mathrm{Si}(\mathrm{Li})$ energy-dispersive spectroscopy (EDS) X-ray detector was the industry standard from the inception of its use on electron microscopes [1]. Since then silicon drift detectors (SDDs) have been introduced [2], and these are currently the $\mathrm{X}$-ray detector of choice across most fields and industries. The original advantage of SDDs was an increase in the speed of the sensor. This "speed gain" was a loosely defined benefit because it referred only to the X-ray charge collection at the chip and the increased rate of electron transfer to the collecting anode due to a voltage-biased drift field. In the earliest generations of SDDs these speed improvements were not fully realized because of limitations in electronic processing and other physical considerations. More recently there have been tremendous improvements in performance of leading-edge SDD technologies at all steps of the signal chain. This has led some companies

(a)
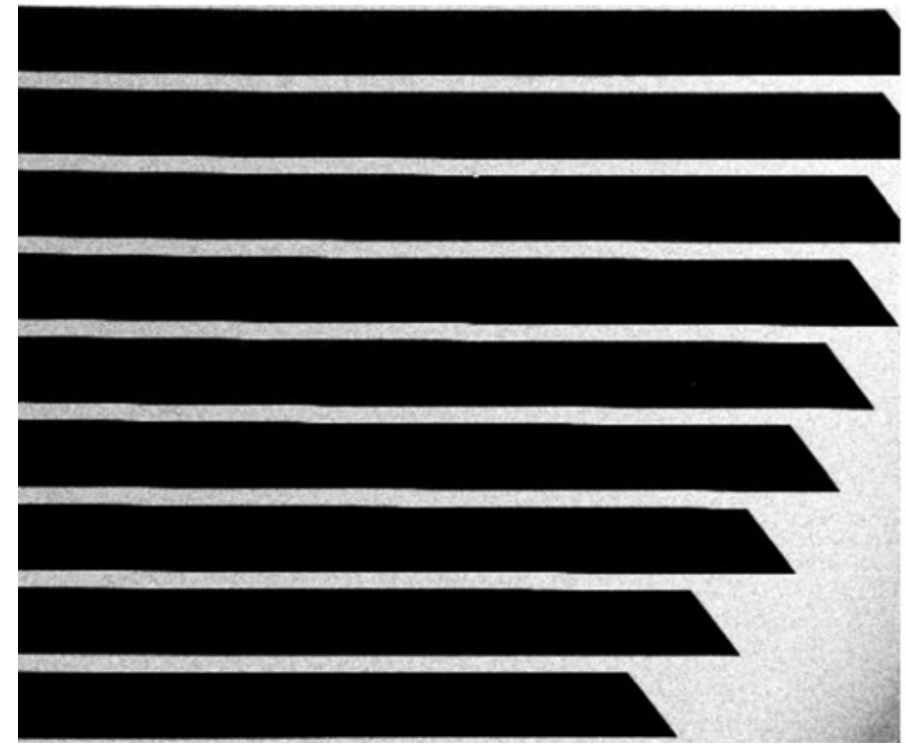

Figure 1: SEM images showing the support grid structure for two X-ray windows. (a) Polymer window. Image width $=2.5 \mathrm{~mm}$. (b) Silicon nitride window. Image width $=1.3 \mathrm{~mm}$. The hexagonal pattern of the silicon nitride window support structure covers $18 \%$ of the window area, whereas the venetian blind support structure for the polymer window covers $23 \%$ of the window area.

to take a whole system approach that provides enhancements in collection efficiency as well as pulse processing speed. In general terms, the final number of useful X-rays analyzed by a given EDS system depends on the number of X-rays generated in the sample, the fraction of the generated X-rays that reaches the detector chip, the detector chip response, and the efficiency of the electronics processing the signal.

\section{Materials and Methods}

Input $\mathrm{X}$-ray count rate. The input counts per second (ICPS) seen by a given EDS detector is highly dependent on the sample, electron accelerating voltage used, and the beam current at the sample. Keeping those parameters the same, the number that is usually considered to have the biggest impact on the ICPS is detector area. While detector area is an easy and intuitive number to understand, one has to be careful when trying to estimate the number of detected X-rays based on this number because the solid angle of the detector can vary significantly depending on which microscope or microscope port the detector is mounted on and how far the detector can be inserted. Recent reports provide solid-angle calculations for different detector geometries [3] and a helpful web-based calculation tool [4].

Window support grid area. A noteworthy parameter in the above-mentioned web-based calculation tool is the areal fraction covered by the window support grid. Most EDS

(b)

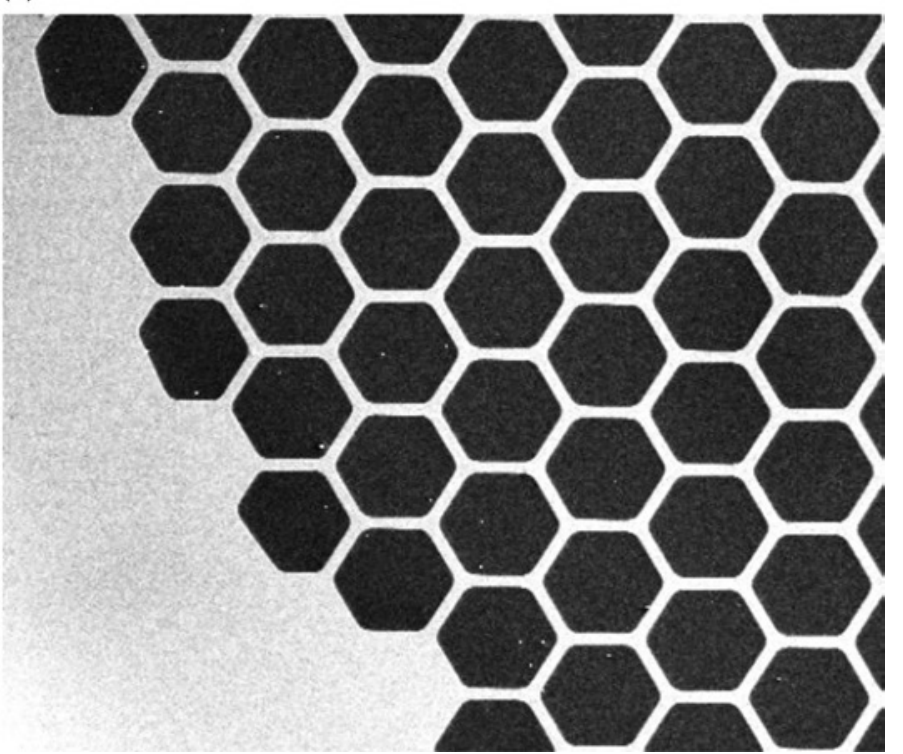




\section{Imaging at sub-nanometer resolution.}

\section{ZEISS GeminiSEM}

// INSPIRATION MADE BY ZEISS
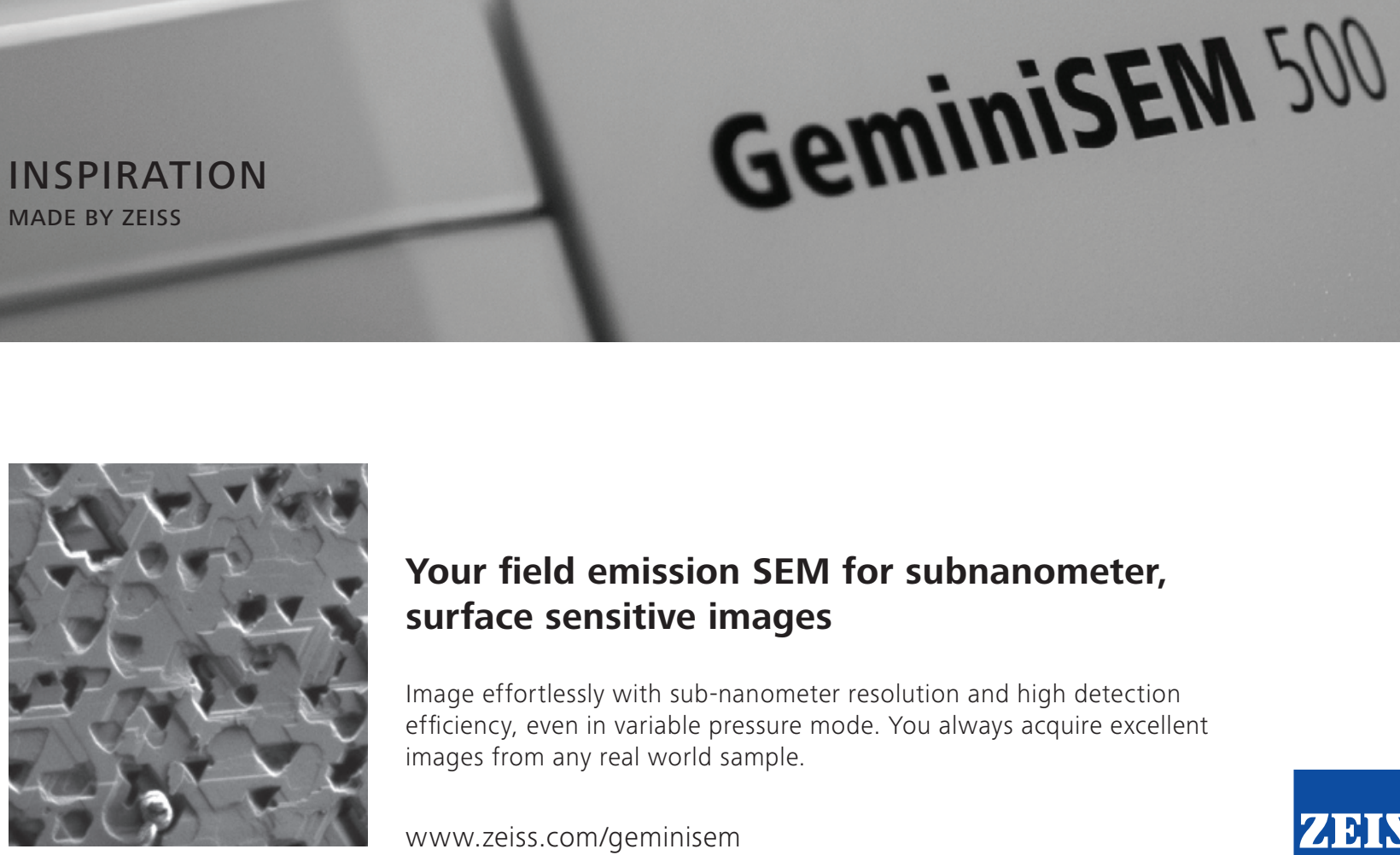

Your field emission SEM for subnanometer, surface sensitive images

Image effortlessly with sub-nanometer resolution and high detection efficiency, even in variable pressure mode. You always acquire excellent images from any real world sample. 


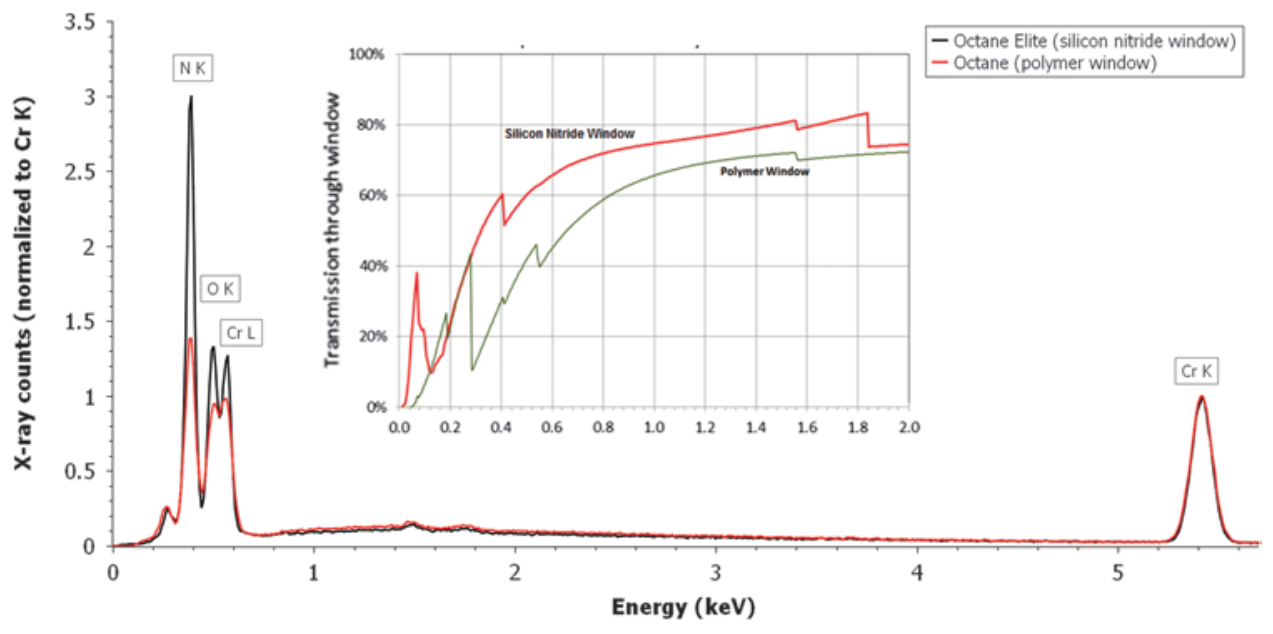

Figure 2: A selection of X-ray element maps acquired from a steel $L$ profile with an average input count rate of 884 kcps at $0.12 \mu \mathrm{s}$ amplifier processing time. (a) Phase map showing steel (yellow) and a Ni-rich inclusion (blue). Inside the Ni-rich inclusion, a Mo-rich phase is shown in bright purple and yellow, and a Si-rich phase is shown in cyan. The epoxy embedding material (green) is carbon-rich. (b) Ni map. (c) Mo map. (d) C map. Image width $=800 \mu \mathrm{m}$.

effects on the nitrogen peak in spectra of $\mathrm{CrN}$.

Output X-ray count rate. With an understanding of the geometric and material aspects affecting the input count rate to the SDD, we can now turn to the relationship of input to output count rate that leads to throughput. The X-ray count throughput is of primary importance to the microanalyst because this is the measure of counts that are actually used in the generation of spectra, linescans, and element maps. The series of processes that describes the ICPS to OCPS conversion is referred to as the "charge-to-voltage conversion." This encompasses (1) the $\mathrm{X}$-ray photon energy conversion to a number of electron-hole pair events

detectors will have a thin window to protect the detector itself from the exterior environment. These windows will have a support grid to prevent the structure from collapsing. Figure 1 shows that the support grid can obscure a significant part of the active area of the EDS detector. Thus, one should consider both the open area of the support grid as well as the active area of the detector when comparing different detector types.

Window material. The composition of the window is another parameter to consider. Earlier systems used Be windows that had very limited transmission in the low-energy range. Current EDS detectors typically have a $300 \mathrm{~nm}$ thick polymer window or a $<100 \mathrm{~nm}$ thick silicon nitride window. Assuming all other parameters are the same (detector-to-sample distance, detector area, support grid coverage), an analysis of a nitrogencontaining sample would result in roughly twice the number of counts in the nitrogen peak for a detector with a silicon nitride window compared to a polymer window. Figure 2 shows transmission curves for the two window materials and their

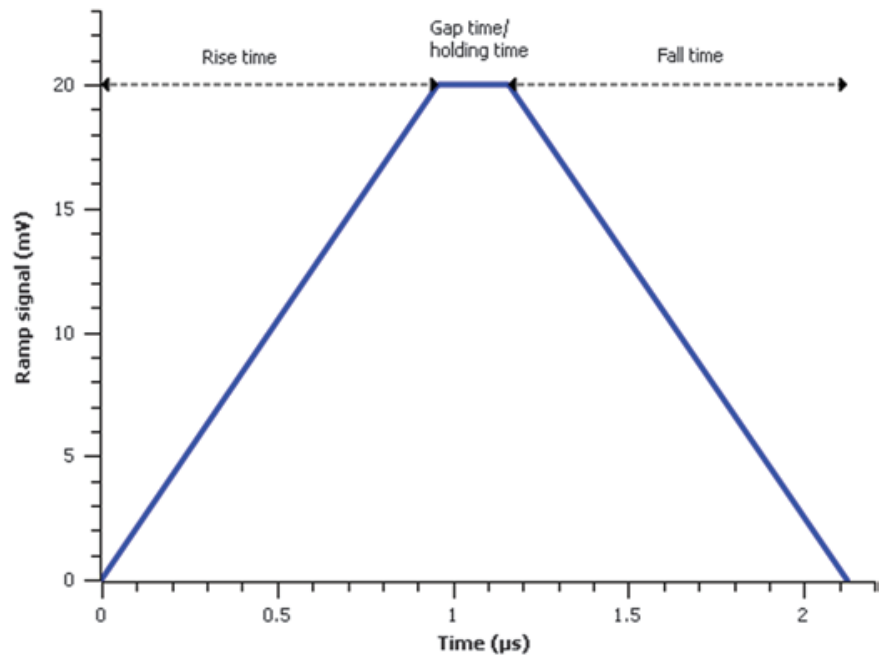

Figure 3: Illustration of pulse processor ramp signal and timings. in the SDD sensor and (2) the charge-to-voltage converter and pulse-shaping amplifier in the electronics [5]. While $\mathrm{Si}(\mathrm{Li})$ detectors commonly had the entire preamplifier external to the detector crystal itself, SDDs often integrate a JFET (junction gate field-effect transistor) on the detector chip to reduce capacitance and noise [6]. Some recent systems employ an external CMOS (complementary metal-oxide semiconductor) preamplifier, which results in further reductions in capacitance and noise, allowing better performance at high count rates and better low-energy response [7]. Following the preamplifier is the pulse processor, which generates a voltage output pulse typically consisting of a triangular or trapezoidal curve with a flat at the top (Figure 3). The total pulse processing time is the amount of time it takes to analyze a single X-ray event and place it in the spectrum; consequently, this factor determines the number of

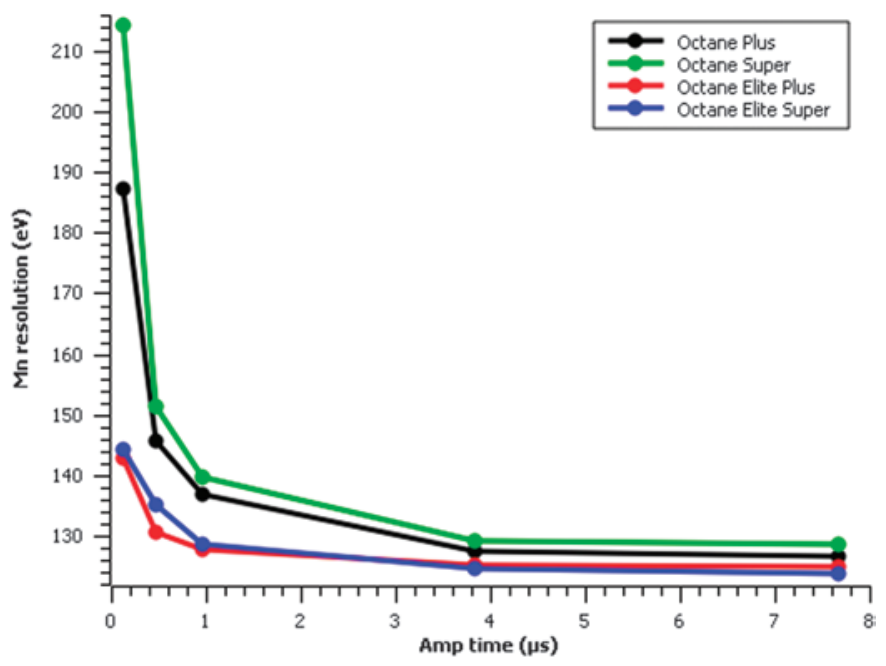

Figure 4: Energy resolution ( $\mathrm{Mn} \mathrm{K} \alpha$ at FWHM) as a function of processing time for two generations and sizes of SDD detectors (Super being roughly twice the area of Plus) where the newer (Octane Elite) employs CUBE technology and shows significant improvements in resolution at fast processing times. The same digital pulse processor was used for all measurements. 


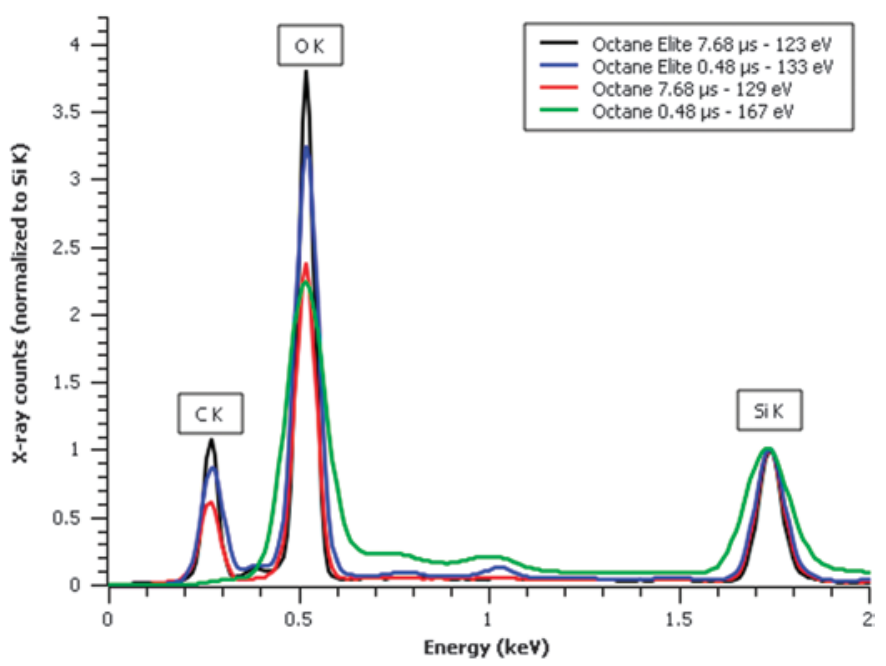

Figure 5: Spectra from a carbon-coated $\mathrm{SiO}_{2}$ sample illustrating energy resolution and low-energy performance at fast processing times for two detectors. The spectra are normalized to the Si K peak. Although these detectors have comparable performance at the slow pulse processing times, the reduced overall noise of the CUBE pre-amplifier results in significantly better energy resolution and low-energy cut-off at fast pulse processing times. Note that $\mathrm{C}$ is not detectable at the $0.48 \mu \mathrm{s}$ processing time in the older generation Octane detector. The increased $\mathrm{O}$ signal for the Octane Elite detector is attributable to the silicon nitride window.

$\mathrm{X}$-rays that the pulse processor can analyze in a given amount of time at different pulse processor settings. Unfortunately, the terminology is not as rigorous as could be desired and often the terms amplification time, processing time, time constant, and peaking time are used interchangeably to either describe the rise/fall time or the entire pulse time. In $\mathrm{Si}(\mathrm{Li})$ detectors the charge cloud had to travel through the bulk of the Si crystal, while the SDD detector utilizes a drift field closer to the surface of the detector, resulting in faster charge collection and thus higher throughput. For SDDs the holding time is typically on the order of a few hundred nanoseconds while the rise/fall time varies from hundreds of ns to tens of $\mu \mathrm{s}$, resulting in total pulse

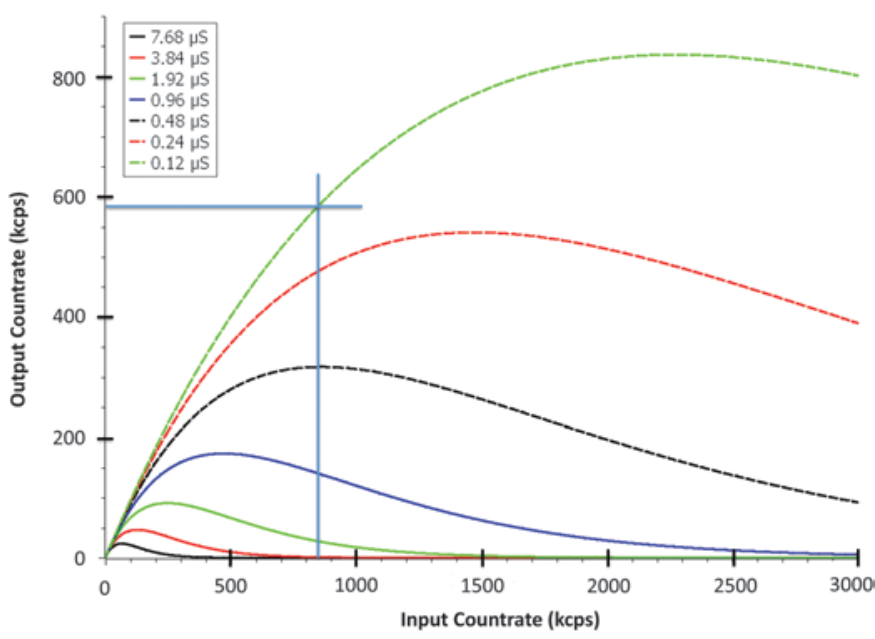

Figure 6: Theoretical SDD output count rate as a function of input count rate for different amplifier processing times. Straight blue lines show that an input count rate of $884 \mathrm{kcps}$ yields $585 \mathrm{kcps}$ output count rate into the spectrum. processing times ranging from roughly $0.5 \mu$ s to several tens of $\mu \mathrm{s}$. The lower limit for the total pulse processing time a given detector can run with, and consequently the upper limit for the throughput, is defined by the amount of time it takes for an electron cloud generated in the detector chip by an X-ray event to move to the detector anode for collection. In order to measure the generated charge correctly, the entire charge cloud has to be collected within the pulse time. If the pulse processing time is shorter than the charge collection time, only part of the energy will be collected within a pulse, resulting in a low-energy tail on the primary peak.

Processing time. An important factor related to the final OCPS is the detector busy time, when the detector system is doing the following: (a) analyzing an incoming pulse, (b) resetting the accumulated charge (reset times are typically less than $1 \mu \mathrm{s}$ ), and transferring data (on newer systems this is done continuously and the detector does not need to stop acquisition to transfer data). When the detector is busy, it should not accept a second X-ray pulse because the system can only process one X-ray event at a time. However, when two X-ray pulses fall within the same processing time window, the energies of the individual $\mathrm{X}$-rays cannot be resolved and both pulses must be ignored. The time elapsed for measuring the discarded pulses is called "dead time." As the ICPS increases, the probability of these near-coincident double pulses also increases and so does the dead time. For a given amp time, a low ICPS registers a low dead time, and a high ICPS yields a high dead time. Thus, the dead time can be used as a relative rate meter.

Throughput versus energy resolution. Many electronics processing architectures provide user selectable "Processing Time" or "Amp Time" settings: short times for high throughput or long times for improved energy resolution to resolve peaks with energies that are closely spaced. Thus, traditionally there was a known trade-off in deciding settings for speed and counting statistics versus energy resolution. The energy resolution of a peak (peak width) is dependent on two factors: the statistical distribution of the charge carrier conversion in the sensor and the thermal noise of the amplification process, primarily from the FET, the first stage of amplification [5]. At short amplifier times there would typically be more noise, leading to peak broadening and a low-energy cut-off as the noise became comparable to the charge contribution of low-energy $\mathrm{X}$-rays. However, recent processing routines using the external CMOS "CUBE" preamplifier significantly reduces the noise at short pulse processing times compared to that of JFET devices [7]. Thus, the trade-off between count throughput versus energy resolution is no longer a factor for EDS systems using CUBE technology. Figure 4 shows representative $\mathrm{Mn} \mathrm{K \alpha}$ energy resolution versus amp time for a selection of detectors, and the improvement at short times for the Octane Elite detectors is evident. Figure 5 shows the improvements in energy resolution and low-energy response for the Octane Elite detector at a short processing time.

Short amplifier processing time is the key to the SDD system's ability to efficiently collect and analyze X-rays at 
high counts rates, rates that were previously impossible. The ultimate throughput of X-ray counts in a system is dependent on the time to process an X-ray pulse (Figure 6) - the shorter the amp processing time, the higher the throughput. Each processing time will have a maximum throughput (the top of each curve in the figure). These high-throughput count rates now permit high-speed $\mathrm{X}$-ray mapping with input count rates over $750 \mathrm{kcps}$. This means the acquisition of a spectrum image that traditionally took hours can now be acquired in minutes.

Instrumentation. The benefits and the improved performance of the new SDD systems can clearly be seen when the advances are applied to typical analytical examples. The data presented here were collected using a JEOL 7000F SEM with an EDAX Octane Elite Super detector with a silicon nitride window

(a)

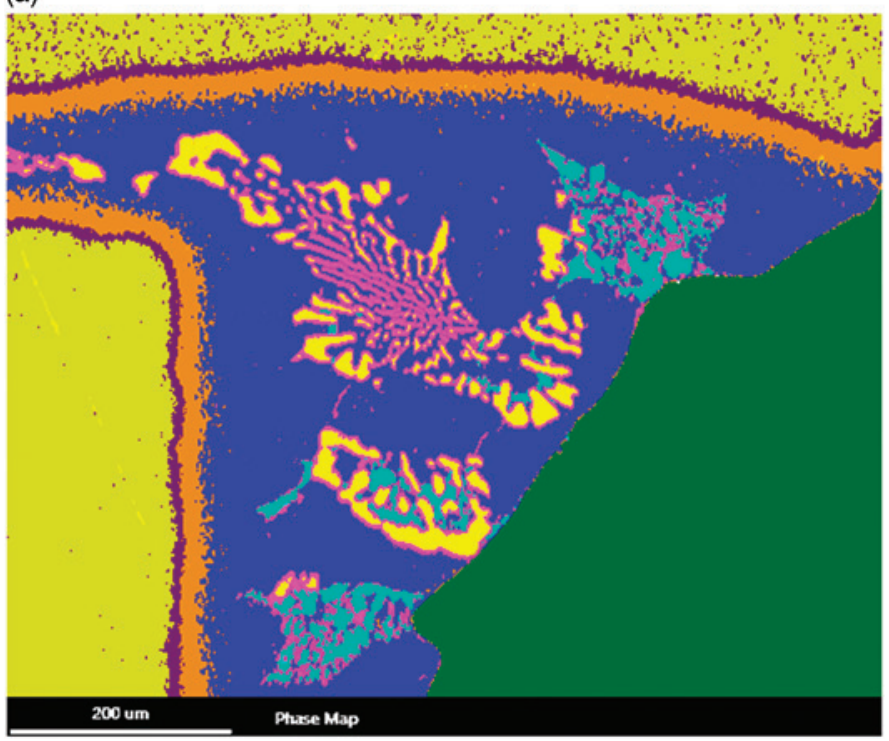

(c)

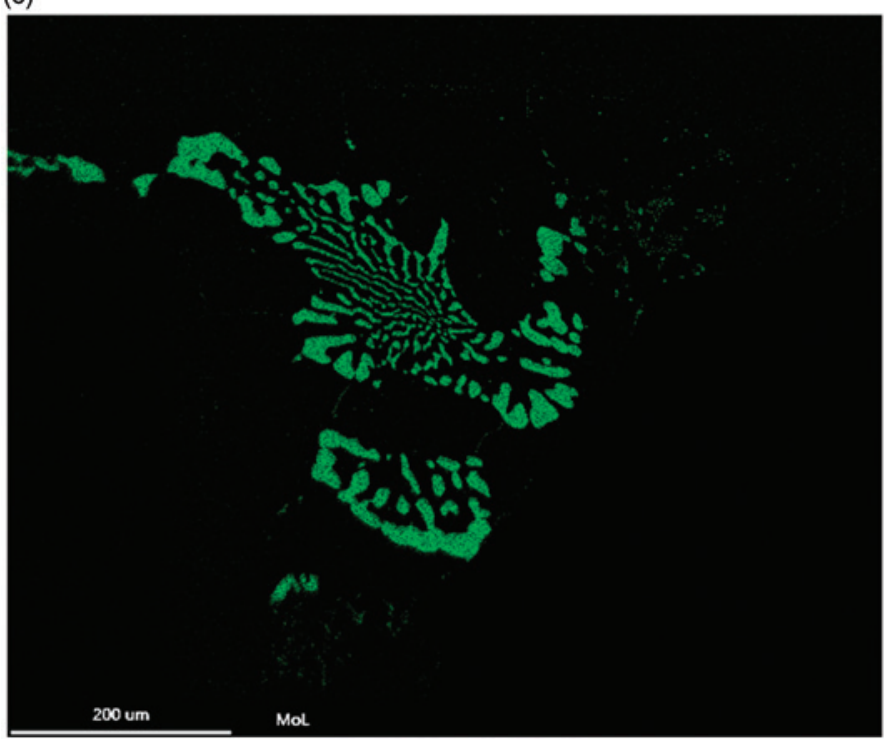

and a CUBE amplifier. To illustrate high-speed mapping, we used an epoxy-embedded, cross-sectioned and polished, cast-steel L profile with compositional variations at the bend of the L. To show quantification reproducibility and light-element sensitivity at high count rates, we present data from a Ti-6Al-4V alloy and a tool steel with a low $\mathrm{C}$ concentration.

\section{Results}

High-speed X-ray mapping. In the past handling high count rates typically meant sacrificing spectral resolution and light-element performance, resulting in data of limited use. However, with modern detectors capable of very high count rates, acceptable qualitative and quantitative data can be acquired in extremely short times. Figure 7 shows a group of $\mathrm{X}$-ray maps acquired with average ICPS of $844 \mathrm{kcps}$ going up

(b)

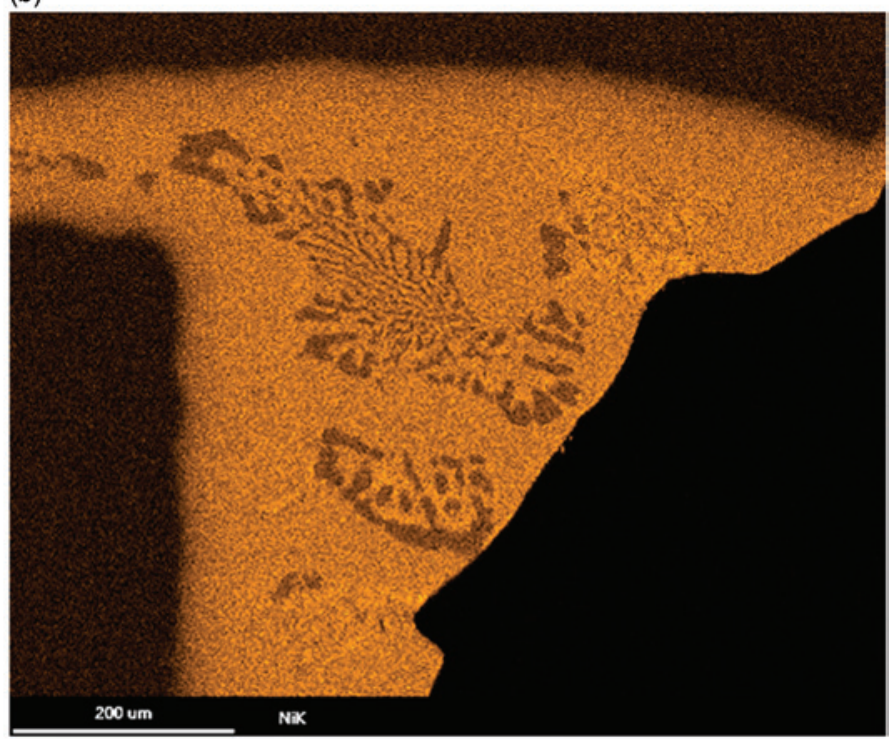

(d)

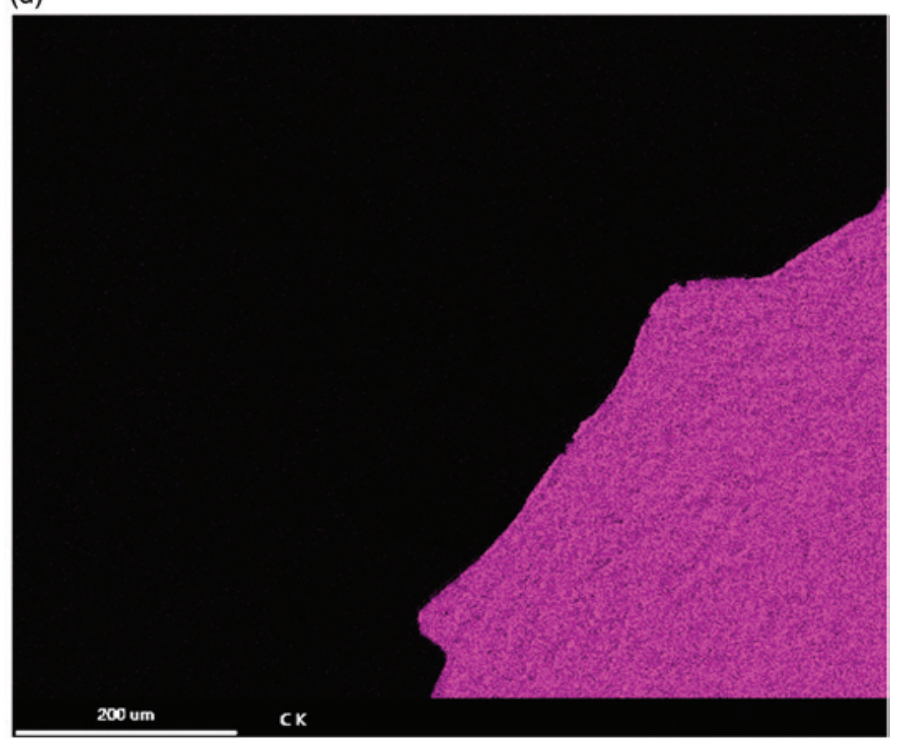

Figure 7: A selection of $X$-ray element maps acquired from a steel L profile with an average input count rate of $884 \mathrm{kcps}$ at $0.12 \mu \mathrm{s}$ amplifier processing time. (a) Phase map showing steel (yellow) and a Ni-rich inclusion (blue). Inside the Ni-rich inclusion, a Mo-rich phase is shown in bright purple and yellow phase, and a Si-rich phase is shown in cyan. The epoxy embedding material (green) is carbon-rich. (b) Fe map. (c) Mo map. (d) C map. Image width $=800 \mu \mathrm{m}$. 


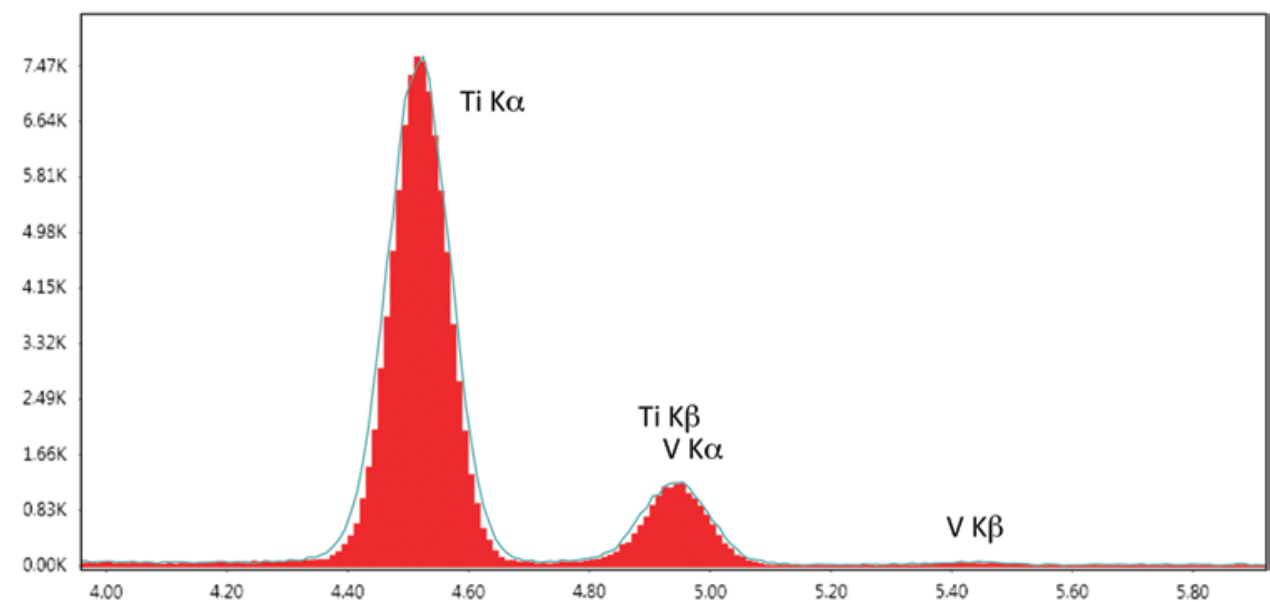

Figure 8: Overlay of normalized spectra collected from a Ti-6Al-4V alloy for 10 seconds at $20 \mathrm{kV}$ and $20,000 \mathrm{cps}$ incoming count rate with a processing times of $3.84 \mu$ s (red solid curve) and $0.24 \mu$ s (cyan outline) under identical microscope conditions. The Ti K $\beta-\mathrm{V}$ K $\alpha$ peak overlap was separated with an iterative Bayesian deconvolution.

quantitative analysis accuracy at high speeds. This alloy has a $\mathrm{Ti}$ $\mathrm{K} \beta-\mathrm{V} \mathrm{K} \alpha$ peak overlap (Figure 8 ), making quantitative analysis of the minor concentration of vanadium a challenge. The overlapped peak separation method in this analysis is based on an iterative Bayesian deconvolution. For more details see [9].

The analysis was performed at traditional collection rates: $20 \mathrm{kV}$, ICPS $=20,000 \mathrm{cps}$, and $30 \mathrm{sec}$ live time. Using an amplifier processing time of $3.84 \mu$ s resulted in a $124 \mathrm{eV}$ energy resolution, and the standardless quantification of the spectrum resulted in weight percent concentrations of $89.39 \% \mathrm{Ni}, 6.17 \% \mathrm{Al}$,

to roughly $1 \mathrm{M}$ cps on parts of the sample. With a short $0.12 \mu \mathrm{s}$ amp time, the average dead time was $37 \%$. For this acquisition a pixel dwell time of $1 \mathrm{~ms}$ was used, which is roughly equivalent to a slow SEM image scan. Even phase maps [8] can be generated with these short dwell and processing times (Figure 7a), while still maintaining sensitivity to low-energy C X-rays. Obviously fast processing times are needed when collecting maps at high count rates, but most of the time, data acquisition is carried out at lower count rates and beam currents, often because of sample limitations or imaging needs. This is where improved detector efficiency can be helpful. For a fixed-input count rate, a better detector efficiency would yield a higher number of counts in the spectrum.

Ti-6Al-4V alloy. The precision and accuracy of quantitative analysis and the software's ability to deal with potential spectral artifacts are concerns with going to higher count rates and faster processing times. To address these concerns, a titanium alloy known as Ti-6Al-4V, a common aerospace alloy known for its superior tensile strength and high-temperature toughness, was used to demonstrate and $4.44 \% \mathrm{~V}$ (Table 1). We will consider these the baseline or "target" quantitative values for subsequent faster collections. Three additional measurements were taken with the same ICPS: one with 10 live seconds collection time at the same processing time and two at $0.24 \mu \mathrm{s}$ processing time with $10 \mathrm{~s}$ and $1 \mathrm{~s}$ collection times, respectively. The quantitative data remained good even for the difficult $\mathrm{V}$ overlap. This shows that the small loss in detector resolution at the short processing times did not affect the quantification reliability, and good quantification is possible even with limited statistics at short collection times. However, to reap the benefits of the faster processing times, one should increase the count rates significantly. Table 1 shows that even at 100,000 ICPS the quantification numbers show no significant changes. An ICPS of 100,000 cps was chosen because this is a value that can be obtained on most microscopes and samples with a reasonable beam current.

Carbon in steel. A second application focuses on the accurate quantification of a known trace amount of carbon in a tool steel standard. Low-energy X-ray analysis of carbon is challenging because of X-ray absorption within the sample and in the various

Table 1: Results of standard-less quantitative analysis of a Ti-6Al-4V alloy. The Ti K $\beta-\mathrm{V} \mathrm{K} \alpha$ peak overlap was separated with an iterative Bayesian deconvolution. Spectrum acquisition was with an Octane Elite Super detector at $20 \mathrm{kV}$.

\begin{tabular}{|l|c|c|c|c|c|}
\hline & \multicolumn{3}{|c|}{ Weight \% } & $\begin{array}{c}\text { Total } \\
\text { Total } \\
\text { collection time }\end{array}$ & $\begin{array}{c}\text { V } \\
\text { spectrum counts }\end{array}$ \\
\cline { 2 - 5 } & $\mathrm{Ti}$ & Al & 4.44 & 36.14 & $723 \mathrm{~K}$ \\
\hline $\begin{array}{l}3.84 \mu \mathrm{s}, 124 \mathrm{eV}, \\
20 \mathrm{kcps}, 30 \mathrm{sec}\end{array}$ & 89.39 & 6.17 & 4.72 & 12.05 & $241 \mathrm{~K}$ \\
\hline $\begin{array}{l}3.84 \mu \mathrm{s}, 124 \mathrm{eV}, \\
20 \mathrm{kcps}, 10 \mathrm{sec}\end{array}$ & 89.09 & 6.19 & 4.66 & 10.20 & $204 \mathrm{~K}$ \\
\hline $\begin{array}{l}0.24 \mu \mathrm{s}, 133 \mathrm{eV}, \\
20 \mathrm{kcps}, 10 \mathrm{sec}\end{array}$ & 89.11 & 6.23 & & & 1.02 \\
\hline $\begin{array}{l}0.24 \mu \mathrm{s}, 133 \mathrm{eV}, \\
20 \mathrm{kcps}, 1 \mathrm{sec}\end{array}$ & 89.06 & 6.05 & 4.89 & $20 \mathrm{~K}$ \\
\hline $\begin{array}{l}0.24 \mu \mathrm{s}, 133 \mathrm{eV}, \\
100 \mathrm{kcps}, 2 \mathrm{sec}\end{array}$ & 89.22 & 6.16 & 4.62 & 2.12 & $213 \mathrm{~K}$ \\
\hline
\end{tabular}


(a)

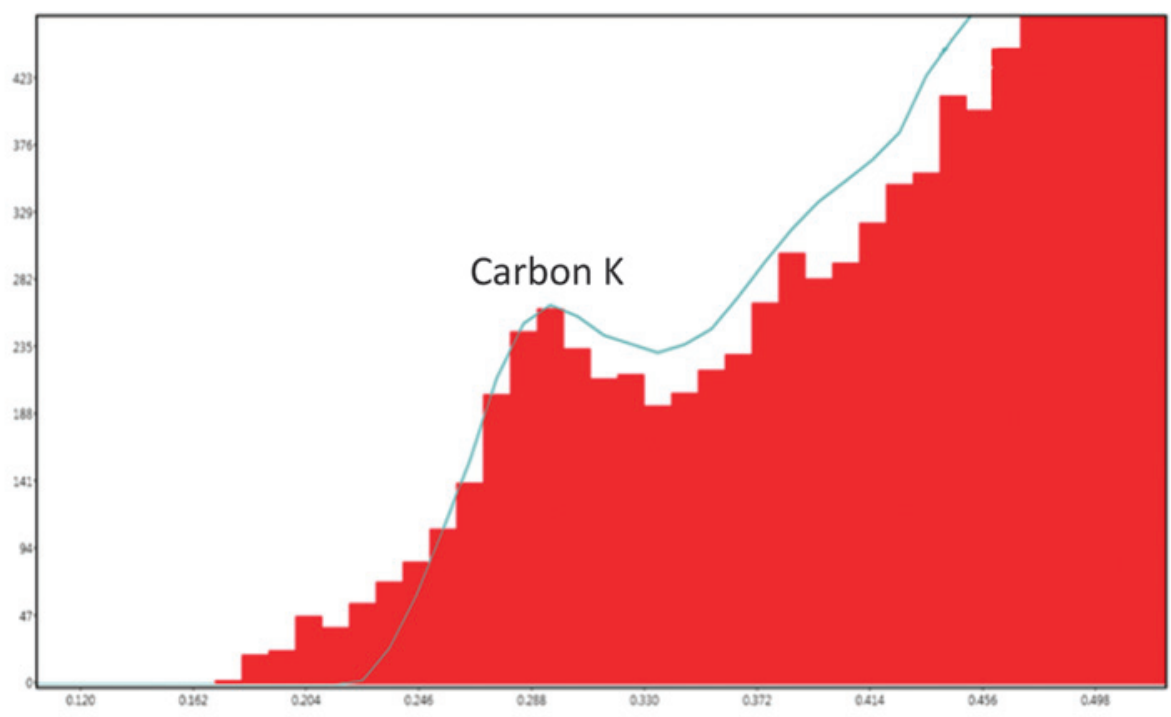

(b)

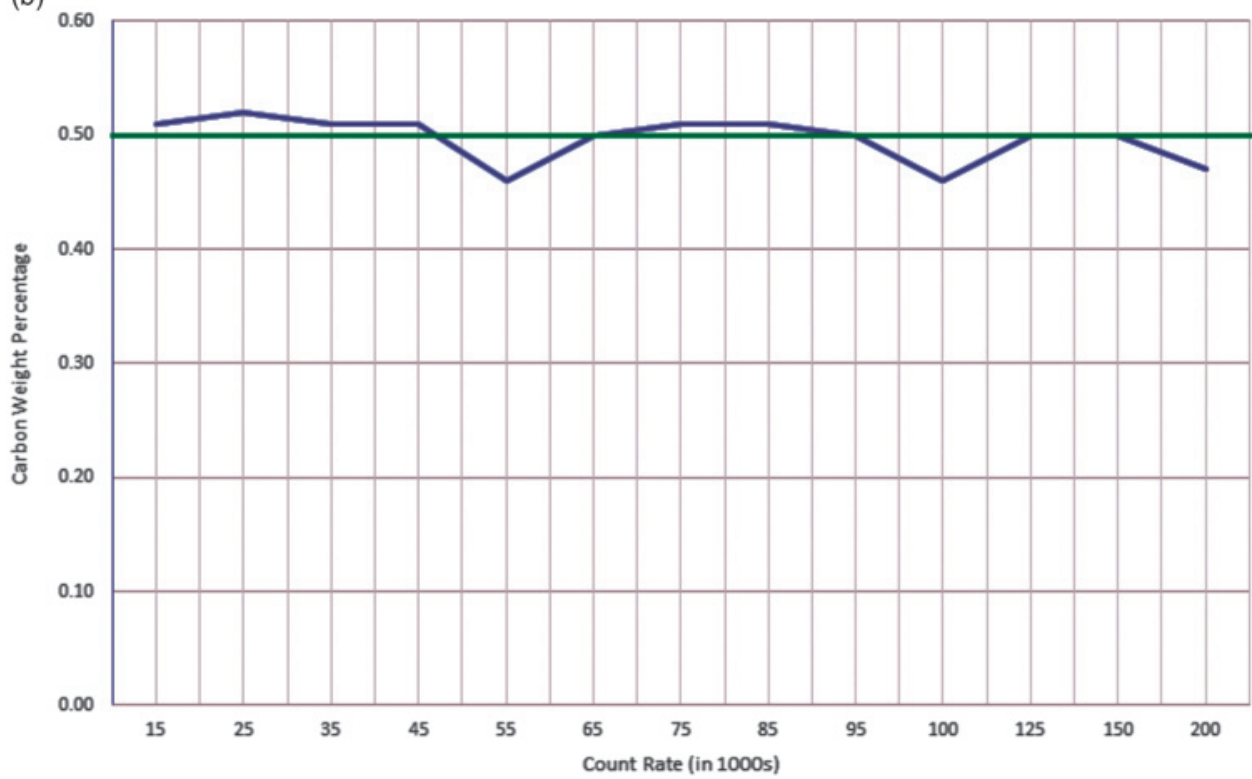

Figure 9: Quantitative results for trace carbon in a steel standard at $20 \mathrm{kV}$ and $30 \%$ dead time. (a) Carbon peak acquired at 15,000 input cps with $7.86 \mu$ s processing time (red) and 200,000 input cps with $0.96 \mu$ s processing time (blue line). (b) Carbon concentration versus input count rate.

components of the detector. The low carbon concentration of $0.50 \mathrm{wt} \%$ creates a further challenge to detection and quantification. In this example, spectra were collected for 30 seconds at count rates ranging from 15,000 ICPS through to 200,000 ICPS, and the amplifier processing time was adjusted to keep the dead time below 30\%. Figure 9a shows an overlay of the zoomed carbon peak region of the spectrum, comparing the two extreme collection rates. Figure $9 \mathrm{~b}$ shows the stability of the results as the count rate was increased, with a $\mathrm{C}$ concentration ranging from $0.46 \mathrm{wt} \%$ to $0.52 \mathrm{wt} \%$ compared with the given known value of $0.50 \mathrm{wt} \%$.

\section{Summary and Conclusions}

In order to really assess the efficiency of an EDS system, one needs to look beyond the simple numbers of detector area or collection solid angle and consider the entire processing chain. The input count rate under a given set of conditions is dependent on geometry, collection solid angle, window material, window support structure, and specific SDD construction. Once the X-rays are detected, they also need to be processed properly to be of any use. With new developments in amplifier circuits and pulse processors, the quality and speed of spectrum acquisition are constantly improving. With improved energyresolution stability and light-element detection at fast processing times, higher throughput can be achieved at modest count rates by using a short pulse processing time with only a slight impact on spectral quality. Fast processing speeds can confidently be used for elemental mapping and for challenging applications with elemental overlaps.

\section{Acknowledgements}

The authors thank Russ Smith of EDAX for his continued focus on the importance of the quality of quantitative results even at high collection speeds. We also thank Kylie Simpson and Robert Rosenthal, our summer interns, who carried out the carbon measurements at high count rates.

\section{References}

[1] R Fitzgerald et al., Science 159(3814) (1968) 528-30.

[2] L Strüder et al., Mikrochim Acta (Suppl. 15) (1998) 11-19.

[3] NJ Zaluzec, Microsc Microanal (2014) 1318-26.

[4] NJ Zaluzec, XEDS Tools Solid Angle Calculator V-2014091607 (October 2016) http:// tpm.amc.anl.gov/NJZTools/XEDSSolidAngle.html.

[5] JI Goldstein et al., Scanning Electron Microscopy and X-Ray Microanalysis, Second edition. Plenum Press, New York, 1992.

[6] P Lechner et al., Adv X Ray Anal 47 (2004) 53-58.

[7] L Bombelli et al., IEEE Nuclear Science Symposium Conference Record (2011) 1972-75.

[8] T Nylese and R Anderhalt, Microscopy Today 22(2) (2014) 18-22.

[9] F Eggert and W Scholz, Phys Status Solidi A 97 (1986) K9.

[10] PT Törmä et al., IEEE T Nucl Sci 61 (2014) 695-9. 


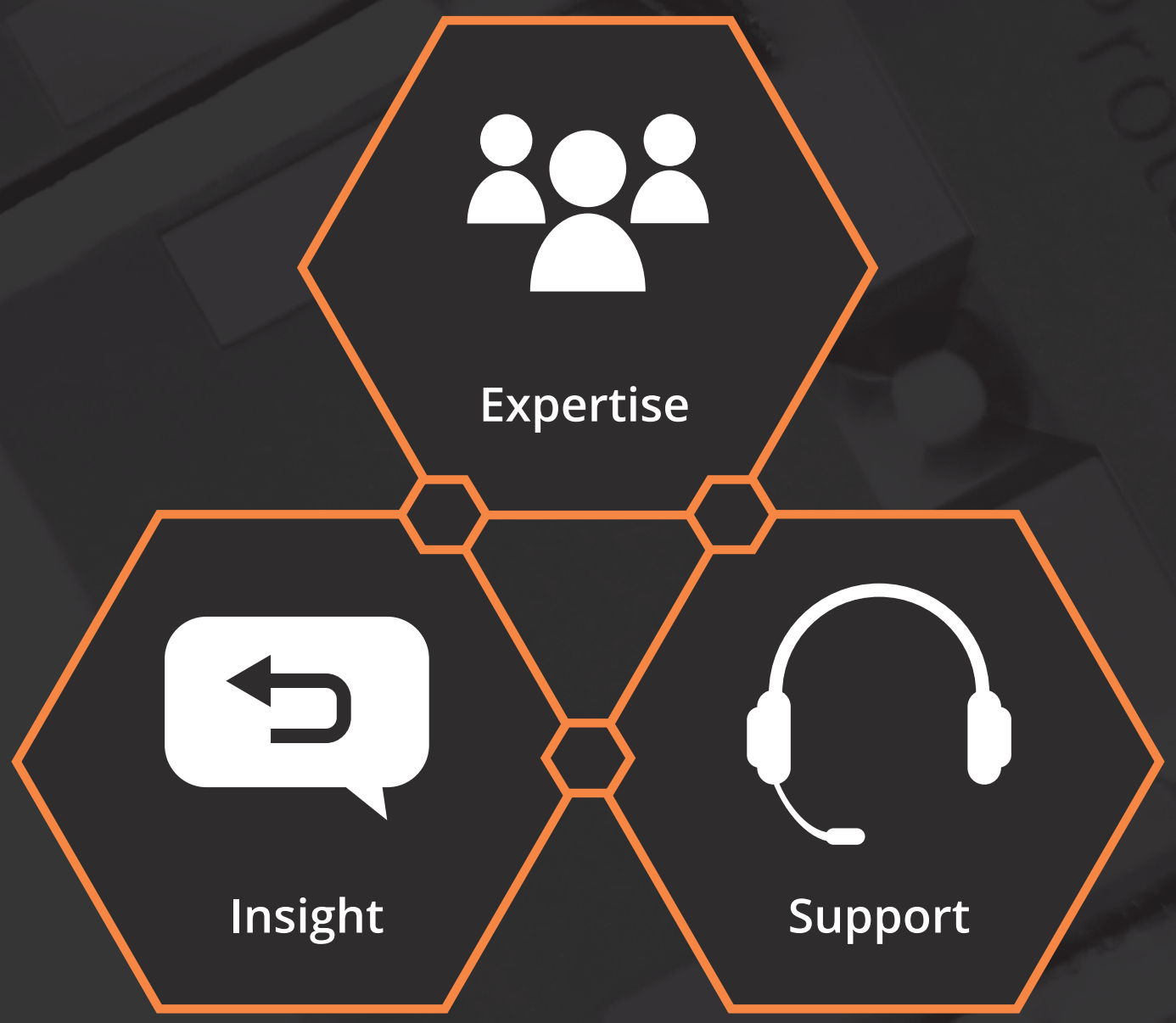

\section{ACCELERATE YOUR IN SITU RESEARCH}

Whether you are researching new materials or discovering new phenomenon in the electron microscope, nothing should delay your experiment. That's why our service team is always ready to help you with topics ranging from product installation and training to troubleshooting and upgrades. And if you need help with an experiment, we have an applications team with decades of in situ experience ready to answer your most challenging questions. We provide all of this for you, because our goal is to accelerate your in situ research. Discover more at: 